\title{
Beryllium abundances in metal-rich stars
}

\author{
Ruth C. Peterson ${ }^{1}$ \\ ${ }^{1} \mathrm{UCO} /$ Lick Observatories and Astrophysical Advances \\ email: peterson@ucolick.org
}

\begin{abstract}
In metal-rich stars as cool as the Sun, beryllium abundance determinations are difficult due to heavy line blanketing in the near-UV $3130 \AA$ region where the accessible Be II lines reside. We can now attempt such determinations based on improved lists of atomic line identifications and gf-values in the near-UV. Here we report Be determinations for three metalrich $\mathrm{A}, \mathrm{F}$, and $\mathrm{G}$ stars plus three solar-metallicity standards. All six stars have beryllium-tohydrogen ratios at or below solar. More such determinations would provide stronger constraints on trends in Be abundance with temperature, metallicity, and age.
\end{abstract}

Keywords. Stars: abundances, individual (HD 61421, HD 72660, HD 165341, HD 179949, HD 217107)

Previous analyses of stellar beryllium abundances have shed light on both the production of beryllium in the interstellar medium, and its destruction in non-standard mechanisms within and among stars. However, except for members of the young Hyades open cluster (age $600 \mathrm{Myr}$ ), existing beryllium abundance determinations are for stars of solar metallicity and lower. Consequently little is known about beryllium production and depletion among old metal-rich stars.

The problem has been the extreme crowding by the rich assortment of spectral lines in the ultraviolet spectra of stars of type F and later. As reported by Peterson et al. (2004) we have empirically improved the lists of optical and near-UV atomic line parameters to the point where near-UV abundance analyses of such stars are tractable.

We first extracted from the ESO UVES and Keck HIRES archives the spectra for two slowly-rotating metal-rich stars from Takeda et al. (2005), HD 179949 (F8.5V) and HD 217107 (G8IV), and for the solar-metallicity standard HD 165341 (K0V; 70 Oph A). We analyzed these together with ground-based spectra of the Sun (G2V) and with space-based HST STIS E230H spectra of two Peterson et al. (2004) Hubble Treasury standards, HD 61421 (F5IV-V; Procyon) and the metal-rich HD 72660 (A1IV).

Beryllium abundances were determined by comparing the observed echelle spectra to calculations run with the Kurucz program SYNTHE and our updated atomic line list, using Castelli \& Kurucz (2003) ODFNEW models. First, the stellar temperature $\mathrm{T}_{\text {eff }}$, gravity $\log g$, and metallicity $[\mathrm{Fe} / \mathrm{H}]$ were found from the optical region of the spectra, except for HD 72660 where the near-UV continuum is seen. Our results confirm Takeda et al.'s parameters for the two cool metal-rich stars. We then matched the entire region near the BeII doublet with a small grid of calculations, to ensure that continuum placement and spectral resolution were determined as reliably as possible. Fig. 1 shows the results of these comparisons.

All stars are found to have a Be abundance of solar or less, including the two cool metal-rich stars. We are unaware of any previous Be determinations in metal-rich stars, nor in A stars. Before concluding that stellar beryllium never exceeds the solar value, more stars need to be analyzed, to understand what role may be played by the destruction of Be in cool or hot metal-rich stars.

This work was supported in part by NASA HST grants GO-9455 and AR-10970. 


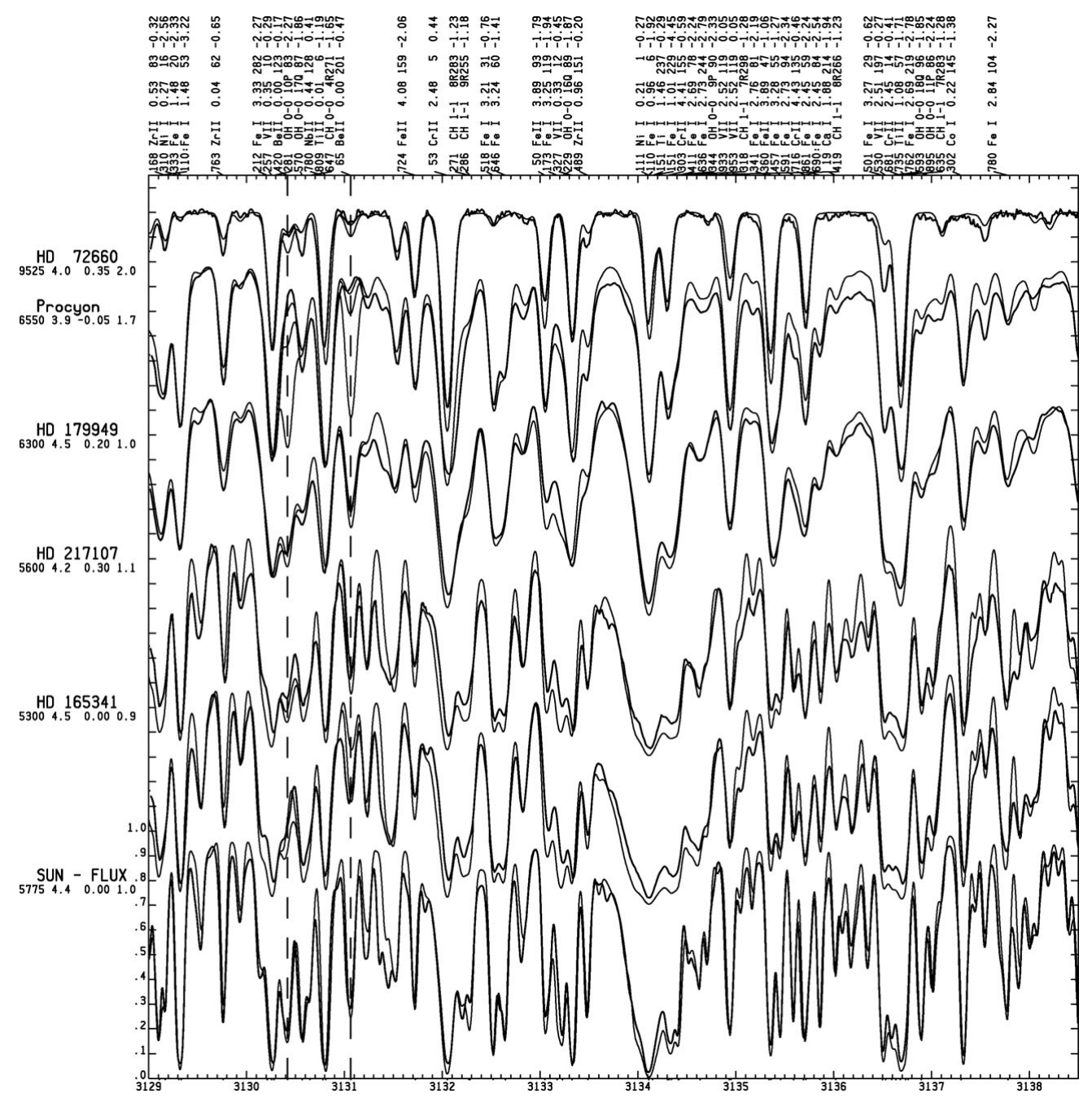

Figure 1. Comparisons between observed spectra (dark lines) and calculated spectra (light lines) are shown in an $11 \AA$ region including the BeII lines. Their positions are indicated by the vertical dashed lines. Each comparison is offset vertically for clarity; ticks denote $10 \%$ of the continuum level. Each star is identified on the left, with the $\mathrm{T}_{\text {eff }}, \log g$, and $[\mathrm{Fe} / \mathrm{H}]$ values of the model used in its calculation. For each star, calculations are shown for two assumed Be abundances: one scaled to the stellar iron abundance, and one adopting the solar Be value itself (half this for HD 165341). For Procyon, which is severely Be-depleted (Boesgaard 1976; Stephens et al. 1997), three calculations show Be abundances of $1 / 10$ and $1 / 40$ solar as well as solar. Identifications of the strongest lines in the calculation appear at the top.

\section{References}

Boesgaard, A. M. 1976, ApJ, 210, 466

Castelli, F. \& Kurucz, R. L. 2003, IAU Symp. No. 210, poster A20; models are at http://wwwuser.oat.ts.astro.it/castelli/grids.html

Peterson, R. C., Carney, B. W., Dorman, B., Green, E. M., Landsman, W., Liebert, J., O'Connell, R. W., Rood, R. T., \& Schiavon, R. P. 2004, STSCI Newsletter, 21, no. 4, p. 1

Stephens, A., Boesgaards, A. M., King, J. R., \& Deliyannis, C. P. 1997, ApJ, 491, 339

Takeda, Y., Ohkubo, M., Sato, B., Kambe, E., \& Sadakane, K. 2005, PASJ, 57, 27 